\title{
Colorectal cancer classifications
}

\section{4}

the cetuximab-

resistant

cell lines

responded to a

MET inhibitor
Clinically distinct subtypes of colorectal cancer (CRC) are starting to emerge through extensive molecular profiling studies. Two new studies have identified different CRC subtypes based on gene expression patterns, and include insight into their possible response to specific therapies.

Sadanandam et al. carried out clustering analysis on published gene expression data from 445 human primary CRC samples. They identified five subtypes based on the expression of 786 genes and named these according to their prominent gene expression signature: gobletlike, enterocyte, transit-amplifying, inflammatory and stem-like. These subtypes were reproducible in seven independent gene expression data sets. Furthermore, four of the five subtypes were found in CRC cell lines, and these were generally

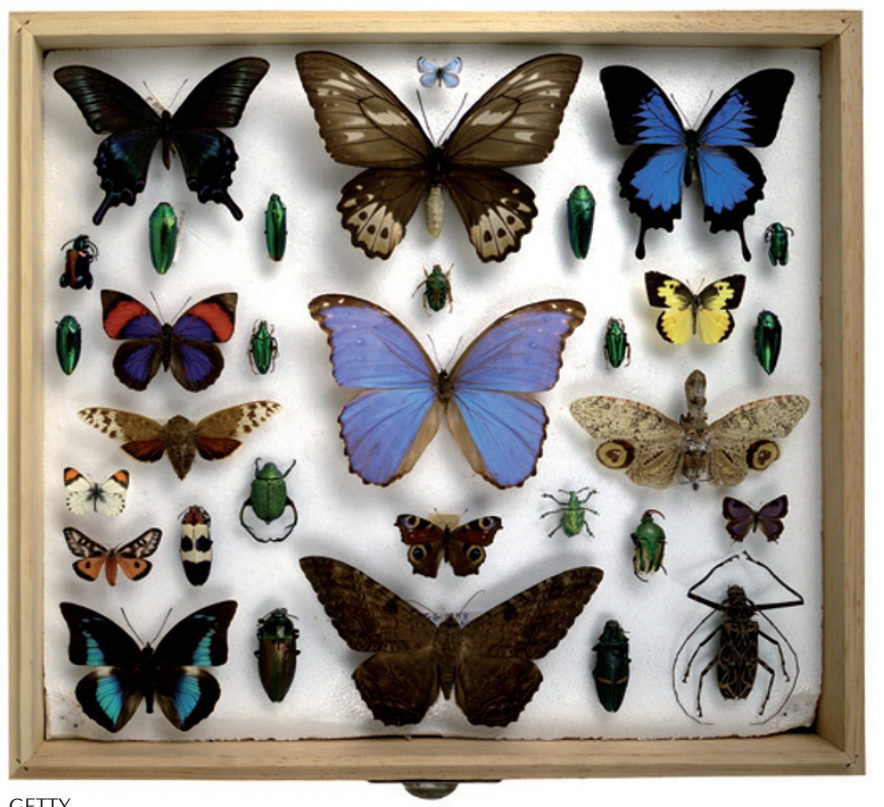

maintained in mouse xenografts of these cell lines, implying that these subtypes are intrinsic to the CRC cells (rather than that they result from the tumour microenvironment) and are fairly stable.

Analyses of the clinical outcomes of patients from whom the samples were obtained revealed the prognostic and predictive values of these classifications. For patients who underwent surgical resection but who were otherwise untreated, the stem-like subtype showed a particularly short time to recurrence. However, this subtype showed the greatest patient benefit from adjuvant chemotherapy. By contrast, the goblet-like and transit-amplifying subtypes were associated with a favourable outcome in patients who underwent surgery alone, but were associated with a poorer outcome in patients who received adjuvant chemotherapy. Thus, these classifiers may inform whether, and how aggressively, different patients should be treated.

Interestingly, the authors found that expression levels of genes - such as the epidermal growth factor receptor (EGFR) ligands epiregulin (EREG) and amphiregulin (AREG), and the MET regulator filamin A (FLNA) - could further divide the transit-amplifying subtype into cetuximab-sensitive versus cetuximab-resistant groups. Crucially, the cetuximab-resistant cell lines responded to a MET inhibitor, suggesting a potential alternative therapeutic strategy.

De Sousa E Melo et al. also carried out gene-expression profiling of human CRC samples. Across a total of 1,100 discovery and validation data sets they clustered CRCs into three subtypes, which were also reproducible and stable in CRC cell lines and xenografts. Interestingly, relative to the subtypes identified by Sadanandam et al., subtype 1 and subtype 2 in this study showed a much stronger concordance with the more traditional subtypes of chromosomal instability (CIN) CRCs and microsatellite instability (MSI) CRCs, respectively. However, subtype 3 is a novel CRC subtype that is mostly microsatellite stable, shows a signature of epithelial-mesenchymal transition and extracellular matrix remodelling, and has a particularly unfavourable prognosis and poor clinical response to cetuximab treatment. Interestingly, this subtype shares gene expression features with the premalignant lesion sessileserrated adenoma, indicating that subtype 3 CRCs may be derived from these adenomas.

Importantly, both teams found that expression analysis of a small subset of genes can be used to classify tumours into their identified subtypes, and they propose that simple assays based on immunohistochemistry or quantitative reverse-transcriptase PCR could be easily integrated into routine clinical practice.

Darren J. Burgess

ORIGINAL RESEARCH PAPERS Sadanandam, A. et al. A colorectal cancer classification system that associates cellular phenotype and responses to therapy. Nature Med. 14 Apr 2013 (doi:10.1038/ nm.3175)|De Sousa E Melo, F. et al. Poor-prognosis colon cancer is defined by a molecularly distinct subtype and develops from serrated precursor lesions. Nature Med. 14 Apr 2013 (doi:10.1038/ nm.3174) 